Proceedings of the 2011 Winter Simulation Conference

S. Jain, R.R. Creasey, J. Himmelspach, K.P. White, and M. Fu, eds.

\title{
MODEL DEVELOPMENT IN DISCRETE-EVENT SIMULATION: INSIGHTS FROM SIX EXPERT MODELERS
}

\author{
Antuela A. Tako \\ Loughborough University \\ School of Business and Economics \\ Loughborough, LE11 3TU, UK
}

\begin{abstract}
This paper reports on an empirical study that explores the model development process followed by six expert modelers in discrete-event simulation (DES). So far the model development practice in DES has not been explored and there is little understanding of the processes followed. This study observes the modeling process of practitioners, experts in simulation modeling undertaking a laboratory modeling exercise. Verbal Protocol Analysis (VPA) is used to collect the data, where the participants are asked to speak aloud while modeling. The data collected are transcribed and a quantitative analysis is undertaken to explore the modeling processes modelers attend to and when during the modeling exercise. The results show that the expert modelers spend a significant amount of time on model coding and verification \& validation. All modelers switch significantly often between different modeling processes. Differences among modelers are observed, which are believed to be attributed to experts' individual modeling style.
\end{abstract}

\section{INTRODUCTION}

Discrete-event simulation (DES) is an established modeling technique within the realm of Operational Research, used widely for decision making. Since the early beginnings of simulation back in the 1950s, a dual relationship between simulation and computing has been noted (Hollocks 2001). Developments in computing such as the increase in computer power, the availability of user-friendly and low budget simulation software has brought about changes in the way simulation models are developed and used (Robinson 2005). One of the main changes noted in the modeling community is a trend of moving away from a large coding effort (Pidd 1999). In addition, simulation modeling has become available to a wider range of modelers, who may not specifically consider themselves experts (Hollocks 2001). Obviously, this has had an impact on the way simulation models are developed. A number of authors have expressed concerns about the misuse of simulation and have touted the importance of following a wider methodology of model development (Pidd 1999; Hollocks 2001; Robinson 2005).

Model development in simulation has been characterized as both art and science (Shannon 1998). A number of models putting forward the steps that should be followed when undertaking a simulation study have been reported in the literature (Balci and Nance 1987; Nance 1994; Pidd 2004; Robinson 2004; Law 2007; Sargent 2010). Other authors provide advice on common pitfalls and modeling principles for others to follow, based on their wide experience as modelers (Shannon 1998; Pidd 1999; Sanchez 2007). Despite this resourceful body of literature providing guidance on the simulation modeling practice, little is known about how simulation models are developed in practice, especially how practitioners go about modeling. Obtaining an understanding of the current practice can in turn serve as a check point between the existing theory and practice of simulation. It can be furthermore useful for teaching simulation to novice modelers.

The aim of this paper is hence to explore the model development process followed by expert simulation modelers. The study undertaken consists of laboratory experiments, where six expert modelers are 
observed while building simulation models of the UK prison population. A qualitative research technique called Verbal Protocol Analysis (VPA) is used to collect the data, where expert modelers are asked to speak aloud their thoughts while they are undertaking a simulation modeling exercise. The data collected in the form of modelers' utterances are analyzed and conclusions are drawn based on expert modelers' live accounts. This study explores mainly the model development process, in the form of the stages followed while undertaking a simulation study and the amount of iterations observed between stages.

The outline of this paper is as follows. Section 2 explores existing references on the model development process, followed by a description of the study undertaken, including the case study, participants and the research method used, Verbal Protocol Analysis (VPA). The data collected are then analyzed providing a quantitative view of the modeling behavior followed by the six simulation experts. Next, the limitations and the main findings of the current study are considered and finally conclusions provided.

\section{THE MODEL DEVELOPMENT PROCESS IN SIMULATION}

\subsection{Models Describing the Model Development Process}

From the beginnings of simulation, the model development process has been described in terms of the stages followed when undertaking a simulation study. Some authors describe this process as models and more specifically life-cycle models, whereas other authors refer to diagrams of the simulation study process. All these models are in principle similar, differing mainly in terms of the names and number of processes or sub-process involved (Robinson 2004). It would be optimistic to name all the existing models in this paper. We will however attempt to refer to some key models identified in the literature. For example Nance (1987 and 1994) developed the so called Conical Methodology, which explained the steps of a simulation study. The work was later updated into a more comprehensive and detailed database, named the Balci-Nance model (Balci and Nance 1987, Arthur and Nance 2007). The authors expanded their work to develop an automated environment with integrated tools to support the modeling process, including among others the specification of model requirements, generation and verification of model code, as well as project management and electronic email system. This model was aimed to assist the modeler especially in developing large and complex models, characteristic of military applications.

An alternative model is the Sargent model (2010), which describes simulation modeling projects from the perspective of integrating verification and validation $(V \& V)$ activities into the model development process. This model considers two separate worlds, the Real World and the Simulation World. These are closely connected between each other in a two-way relationship. Initially abstraction and hypothesizing about the Real World takes place, leading to defining System Theories that are next taken further into a set of modeling phases in the Simulation World. The results obtained from the simulation model are next used to hypothesize about the Real World. This implies that a phase of problem understanding is essential before building a simulation model. The framework furthermore maintains that the process of developing valid models requires an iterative approach between modeling stages.

Other authors, including Shannon (1975), Banks et al. (2001), Law (2007), Pidd (2004) and Robinson (2004) describe the generic activities followed when building simulation models. The key activities included are: problem structuring, conceptual modeling, model coding, experimentation, implementation and validation and verification. A brief description of the activities involved is next provided.

- Problem structuring is concerned with understanding of the issues to be addressed by the project. Pidd (2004) introduces problem structuring as a separate phase from conceptual modeling.

- Conceptual modeling is about identifying what and how to model. This activity is different to problem structuring in that, the problem already identified is now considered in more detail with regards to determining problem objectives, design of the communicative model, identifying assumptions and simplifications and data collection.

- Model coding is concerned with converting the conceptual model into a computer model.

- Experimentation deals with the model results obtained, undertaking experiments searching for potential solutions and sensitivity analysis. 
- Implementation is concerned with the benefits sought from the study, in the form of specific recommendation or improved knowledge and insight. It can take three different forms or a combination of thereof, implementing the findings of the simulation model, implementing the simulation model itself and/or implementing the learning or insights gained from the model developed (Robinson 2004).

- Verification and validation $(\mathrm{V} \& \mathrm{~V})$ consists of actions taken throughout the modeling process to gain confidence in the model and its results.

Other authors provide advice on common pitfalls and modeling principles for others to follow, based on their wide experience as modelers (Shannon 1998; Pidd 1999; Sanchez 2007). Pidd (1999) for example refers to the importance of learning the modeling skills as compared to learning about models. Shannon (1998) furthermore lays out the "40-20-40 Rule", referring to the proportion of time spent among 12 modeling activities that the author considers. According to Shannon, $40 \%$ of the effort and time of a project should be devoted to problem definition, project planning, conceptual modeling and data collection, $20 \%$ to model coding and the remaining $40 \%$ to $\mathrm{V} \& \mathrm{~V}$, experimentation and reporting.

\subsection{Empirical studies of Model Development}

A limited number of papers undertake a more empirical view into the modeling process. Cochran, Mackulak, and Savory (1995) asked practitioners to divide total project effort among the simulation phases. Respondents estimate that data acquisition is the most time-consuming activity, requiring almost $15 \%$ of total project effort. The next most time consuming phases were reported to be problem formulation, model translation, and model building. In a study focusing on experimentation methods used in simulation, Hollocks (2001) interviewed four individual modelers acting as consultants in major organizations. Among others the interviewees were asked to estimate the amount of time spent on different modeling activities in their organization, specified as: pre-modeling, modeling, experimentation and postexperimentation. A general consistency was reported with approximately $40 \%$ of time spent on modeling and $20-30 \%$ on experimentation. The individual answers are displayed in Table 1 below.

Table 1: Proportion of time spent on modeling activities as reported in Hollocks (2001)

\begin{tabular}{|l|c|c|c|c|}
\hline Modeler & Pre-modeling & Modeling & Experimentation & Post-experimentation \\
\hline 1 & $20 \%$ & $40 \%$ & $20 \%$ & $10 \%$ \\
\hline 2 & $15-20 \%$ & $35 \%$ & $30 \%$ & $15-20 \%$ \\
\hline 3 & $20 \%$ & $40 \%$ & $20-30 \%$ & $10-20 \%$ \\
\hline 4 & $5 \%$ & $80 \%$ & $10 \%$ & $5 \%$ \\
\hline
\end{tabular}

The study by Wang and Brooks (2007) is by far the only study that observes the modeling process followed by novices (students) and one expert while undertaking real life modeling exercises. The data were collected by asking the participants to record the time spent on the different modeling activities as they were building models. Even though the study concentrated on conceptual modeling it mapped out the amount of time spent by the expert modeler and novice modelers in different modeling activities. Even though the activities undertaken by the expert modeler were approximated to match the novice modelers reports, comparing the expert modeler's activity to that of novices the study found that the expert modeler spent more time in conceptual modeling, model coding and verification and validation, whereas novice modelers spent a higher proportion of time on data collection and report writing.

Summarizing on the above, it is identified that formal models outlining the model development process have been developed in the literature. However, empirical work dedicated to exploring the modeling process undertaken in practice is limited. The only study that followed modelers (an expert and 9 novices) while building real life models was that reported by Wang and Brooks (2007), focusing primarily on conceptual modeling aspects of a simulation study. It can be argued that this study relied on participants' reports of the time spent on different modeling activities and furthermore only 1 expert modeler took part in 
the study. Hence in order to explore the practice of model development, a study with six expert modelers is undertaken in this paper, where expert modelers' thoughts are recorded while building a simulation model. The overall objective of this study is to explore empirically the model development process followed by expert modelers as they build simulation models. It is expected that expert modelers will attend to the different modeling processes throughout a modeling project, in an iterative way as it has been already found out about general OR modeling by Willemain (1995).

\section{METHODS}

In this section the study undertaken is explained. First, the modeling exercise will be briefly described, followed by a brief introduction to the research method used that of verbal protocol analysis (VPA). Next, we report on the profile of the participants involved in the study and the coding process carried out.

\subsection{The Modeling Exercise}

The modeling exercise used as the stimulus for inducing modelers' thoughts asked modelers to build models of the UK prison population, based on a study reported by Grove, Macleod, and Godfrey (1998). The UK prison population problem was considered suitable because it is sufficiently general and broad to enable the development of a relevant model in a limited time period of around 60 minutes. The time limitation was necessary to ensure participation of simulation practitioners, with busy time schedules. The topic is furthermore a topical problem in the UK, often receiving media coverage, hence it is reasonably familiar to most participating simulation experts. Furthermore, this case study has been already used as part of the author's research on the comparison of DES and SD (Tako 2009).

A case study was set out, providing a brief introduction to the prison population problem with particular attention to the issue of overcrowded prisons. Descriptions of the reasons for, and impacts of, the problem are also provided, including figures and facts, most of which are based on reality, but slightly adapted for the purposes of the research. Two types of prisoners are considered, petty and serious offenders. There is already an initial number of prisoners in the system $(76,000)$. Offenders enter the system as first time offenders and receive a sentence depending on the type of offence. Petty offenders enter the system at a higher rate, on average 3,000 people/year vs. 650 people/year for serious offenders, but receive a shorter sentence length, on average 5 years vs. 20 years for serious offenders. After serving time in prison the offenders are released. A proportion of the released prisoners re-offend and go back to jail (recidivists) after on average 2 years. Petty prisoners are more likely to re-offend. For more details on the case study the reader is referred to (Tako and Robinson 2010).

References to two possible courses of action, in terms of scenarios are made, either to increase the current prison capacity and so facilitate the introduction of stiffer rules, or the alternative of reducing the size of the prison population by introducing alternatives to jail and/or enhancing the social support provided to prisoners. Participating modelers were asked to build a simulation model to be used as a decision-aiding tool for policy making.

\subsection{Verbal Protocol Analysis (VPA)}

VPA is a research method derived from psychology. It requires the subjects to 'think aloud' when making decisions or judgments during a problem-solving exercise. It relies on the participants' generated verbal protocols in order to understand in detail the mechanisms and the internal structure of cognitive processes that take place (Ericsson and Simon 1984). Therefore, VPA as a process tracing method provides access to the activities that occur between the onset of a stimulus (case study) and the eventual response to it, that is model building (Ericsson and Simon 1984, Todd and Benbasat 1987). Willemain (1994 and 1995) was the first to use it in Operational Research (OR) to document the thought processes of OR experts while building models.

VPA is considered to be an effective method to study the model development process in simulation modeling. It is useful because of the richness of information and the live accounts it provides on the ex- 
perts' modeling process. It furthermore overcomes problems related with alternative methods such as interviews or questionnaire surveys, where data collected rely on respondents' reflections on their modeling which may not reflect the practice truthfully. This can be either because they rely on respondents' memory of how they go about modeling or due to the desire of conforming to the generally accepted rules. VPA is used to capture modelers' thoughts in practical modeling sessions in a controlled experimental environment, using the UK prison population modeling exercise, already described, as the common stimulus.

Protocol analysis as a technique has its own limitations. The verbal reports may omit important data (Willemain 1995) because the experts being under observation may not behave as they normally would.

The modelers are asked to work alone and this way of modeling may not reflect their usual practice of model building, where they would interact with the client, colleagues, etc. In addition, there is the risk that participants do not 'verbalize' their actual thoughts, but are only 'explaining'. To overcome this and to ensure that the experts speak their thoughts aloud, short verbalization exercises, based on Ericsson and Simon (1984) were run at the beginning of the sessions.

\subsubsection{The Modeling Sessions}

The expert modelers involved in this study were given the prison population exercise at the start of the session and were asked to build simulation models using their preferred simulation software. During the modeling process experts were asked to 'think aloud' as they model. The researcher sat in the same room, but social interaction was limited. She only intervened in the case that participants stopped talking for more than 20 seconds to encourage them to "keep talking". Explanatory questions were also answered. The modeling sessions were held separately with each individual modeler, in an office environment. The sessions lasted approximately 60-90 minutes. The participants had access to writing paper and a computer with relevant simulation software. The protocols were recorded and later transcribed.

\subsection{The Participants}

The subjects involved in the modeling sessions were 6 simulation experts in DES modeling. All participants use simulation modeling as part of their work, most of them holding consultant positions in different organizations. The companies they come from are established simulation software companies or consultancy companies based in the UK. Involving practitioners, who apply simulation modeling mainly in consultancy projects, rather than academics, was considered appropriate given that this research is concerned with the practical aspect of simulation modeling as opposed to the theory behind it. According to Todd and Benbasat (1987), due to the richness of data found in one protocol, VPA samples tend to be small, between two to twenty. Hence, the sample size of 6 participants is considered reasonable. Table 2 provides a list of participants' profiles.

Table 2: Profiles of participating expert modelers

\begin{tabular}{|c|c|c|c|}
\hline Modeler & $\begin{array}{c}\text { Modeling } \\
\text { experience }\end{array}$ & $\begin{array}{c}\text { Simulation } \\
\text { software }\end{array}$ & $\begin{array}{c}\text { Education on } \\
\text { Simulation } \\
\end{array}$ \\
\hline 1 & 9 years & Witness & On the job \\
\hline 2 & 4 years & Simul8 & At university + job \\
\hline 3 & 13 years & Flexsim & On the job \\
\hline 4 & 8 years & Simul8 & On the job \\
\hline 5 & 4 years & Witness & At university + job \\
\hline 6 & 8 years & Witness & At university + job \\
\hline
\end{tabular}


For reasons of confidentiality participants' names are not revealed. Their names are disguised, where each participant is given a number, hence called modeler 1,2,3, etc. The six expert modelers who participated in the study had a mixture of backgrounds, having completed either doctorates or masters' degrees in engineering, computer science, Operational Research or held MBAs. Their experience in simulation modeling ranges between 4 and 13 years, three of them having been introduced to simulation through their university degrees, whereas the other three were introduced to simulations modeling as part of their jobs. They boast an extensive experience of modeling in areas such as: NHS, criminal justice, food \& drinks sector, supply chain, etc. Participants could use their preferred simulation software. As a result 3 participants used Witness, 2 Simul8 and only 1 Flexsim. Admittedly a more even spread of simulation software among modelers would have been preferred, however at the time of data collection these were the main UK contacts available to the researcher. Nevertheless, the sample chosen provides some level of diversity among the participants in terms of length of modeling experience and simulation software used.

\subsection{Coding the Protocols}

A coding scheme was devised in order to identify what the modelers were thinking about in the context of simulation modeling. The coding scheme was devised following the stages of typical DES and SD simulation projects, based on Robinson (2004), Law (2007) and Pidd (2004). The definition of each modeling topic and aspects covered is provided in the list below:

1. Problem structuring (PS): What is the problem? What are the objectives of the project?

2. Conceptual modeling (CM): Is a conceptual diagram drawn? What are the parts of the model? What should be included in the model? How to represent people? What variables are defined?

3. Model coding (MC): What model components are entered in the model? How is the initial condition of the system modeled? What units (time or measuring) are used? Does the modeler refer to documentation? How is the user interface modeled?

4. Data inputs (DI): How are the already provided data used? Are modelers interested in randomness? How are missing data derived?

5. Model results \& experimentation (EXP): What are the results of the model? What sort of results the modeler is interested in? What scenarios are considered?

6. Implementation (IMPL): How will the findings be used? What learning can be achieved?

7. Verification \&Validation (VV): Is the model working as intended? Are the results correct? How is the model tested? Why is the model not working?

After each modeling session was completed the audio-recorded verbal protocol was transcribed. Each transcript, consisting of one verbal protocol was next divided into episodes or 'thought' fragments, where each fragment is the smallest unit of data meaningful to the research context. Then each episode was coded into one of the 7 modeling topics or an 'other' category for verbalizations that were not related to the modeling task. Some episodes still referred simultaneously to 2 modeling topics and, therefore, were given two modeling topics. The same coding scheme as in Tako and Robinson (2010) was used, where also a pre-test with 4 academics was initially undertaken to test the codes.

The transcripts were coded manually using a standard word processor. According to Willemain (1995), the coding process requires attention to the context a phrase is used in and, therefore, subjectivity in the interpretation of the scripts is unavoidable. In order to deal with subjectivity, multiple independent codings were undertaken in two phases. In the first stage, the transcripts were coded twice with a gap of 3 months between codings. Overall, a 93\% agreement between the two sets of coding was achieved, which was considered acceptable. The differences were examined and a combined coding was reached. Next, the coded transcripts with the combined codes were further blind checked by a third party, knowledgeable of OR modeling and simulation. The differences were discussed and re-examined by both parties to arrive at a consensus coding. Overall, a 90\% agreement between the two codings was achieved. The results from the coded protocols are next presented and discussed. 


\section{ANALYSIS OF PROTOCOLS}

This section provides a quantitative description of the six participants' modeling behavior. The analysis explores the distribution of attention among modeling topics, the sequence of modeling topics attended to during the model building exercise and the pattern of iterations followed among topics. The findings from each analysis follow.

\subsection{Attention Paid by Modeling Topic}

To explore the distribution of attention to the seven modeling topics, the number of words articulated by modelers is used to measure the amount of verbalization. In turn, this is used to indicate the spread of modelers' attention among the modeling topics. Figure 1 shows the proportion of total words verbalized under each modeling topic by the six modelers. The modelers involved in this research concentrated their attention mostly on model coding, verification \& validation and data inputs, by devoting $42 \%, 21 \%$ and $16 \%$ of their attention respectively. The attention paid on experimentation is relatively lower. A more detailed analysis of the episodes on experimentation shows that modelers' attention concentrates mainly on exploring model results rather than on setting up alternative scenarios. Whereas the amount of verbalizations for problem structuring and implementation is negligible.

The data displayed in Figure 1, show a smaller attention paid to conceptual modeling, compared to the expert modeler followed in the study undertaken by Wang and Brooks (2007). Obviously this data reports on a relatively larger sample (of six expert modelers), however this discrepancy could be because the modeling exercise provided a description of the situation, hence the modelers needed to think less about what needed to be modeled. On the other hand the study of Wang and Brooks (2007) used a different method of collecting the expert data, where the expert modeler, recorded the time spent on the different activities himself/herself on a weekly basis. Hence the data may not be directly comparable.

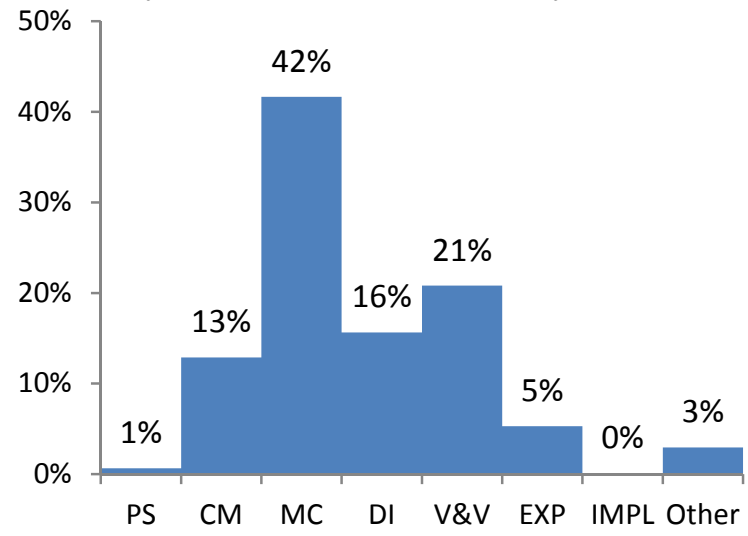

Figure 1: Proportion of attention paid by modeling topic by the six modelers

Figure 2 maps out the number of words articulated by each modeler for the 4 most popular modeling topics (CM, MC, DI, V\&V) in a radar diagram. Each modeler is represented in a different color. As already concluded by exploring the proportion of attention above, all modelers spend most of their time on model coding, which results in a skewed diamond-shape towards the model coding axis. Considering the diamond shapes for each modeler in more detail, two significant patterns of modeling behavior can be identified. The most skewed shapes are evident for modelers 3 and 5, who used Flexsim and Witness respectively. Eliminating modelers 3 and 5 (graph on the right hand side in Figure 2), it can be observed that modelers 2 and 4 have a more symmetrically divided modeling activity among the 4 modeling topics and more specifically the shapes are less skewed, as a comparatively equivalent attention is paid on verification and validation as on model coding. The modeling activity undertaken by modelers 1, 3, 5 and 6 concentrates significantly on building the computer model depicted by a largely skewed diamond shape 
towards model coding. Considering these modeling profiles, two different patterns of modeling behavior are evident, which seems to be due to the modeler's personal style rather than due to the software used or the modeler's length of experience or their education vs. experience on simulation.

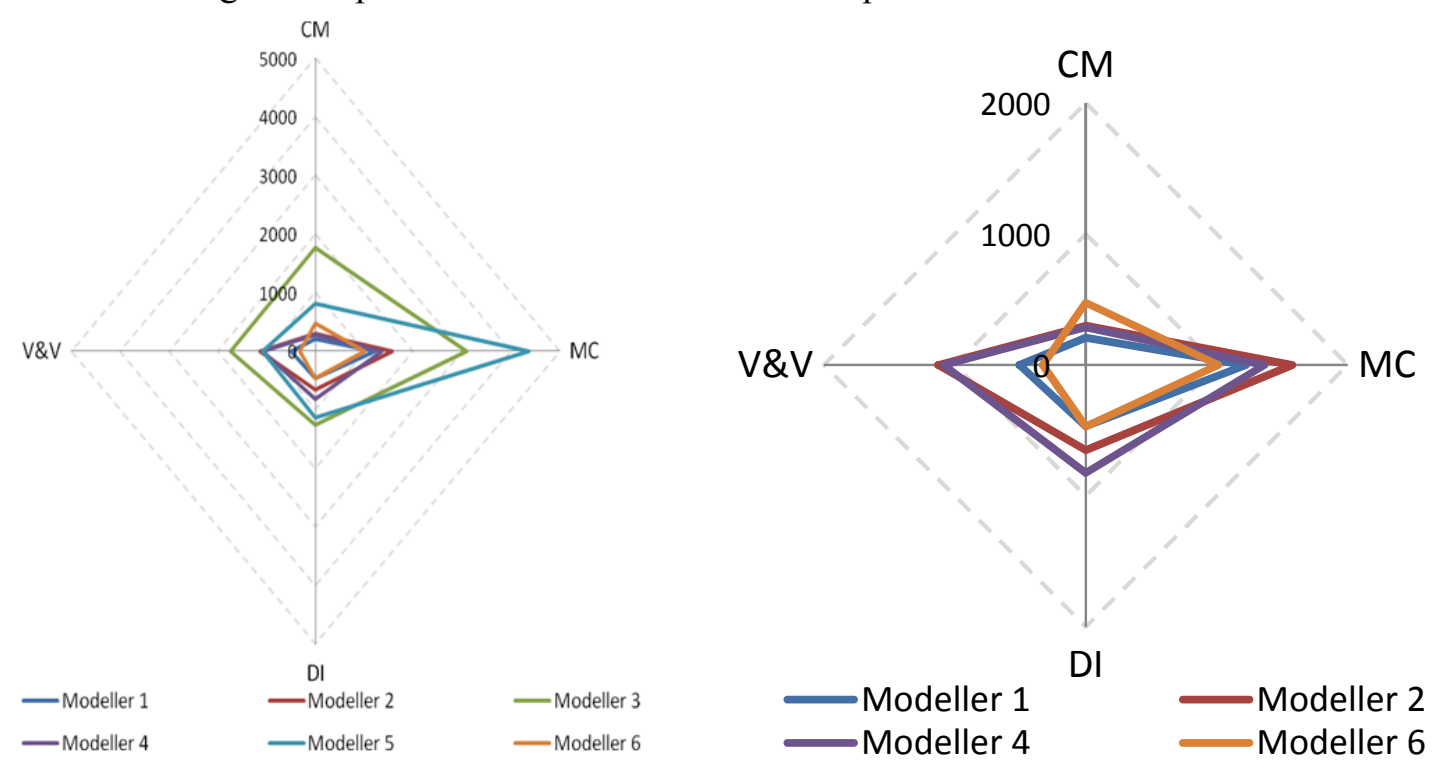

Figure 2: Radar diagrams displaying the number of words verbalized for the four most common modeling topics (Conceptual modeling, Model coding, Data inputs and Verification \& Validation) by modeler. The diagram on the left displays data for all six modelers, data for modelers 3 and 5 are omitted from the diagram on the right.

\subsection{Sequence of Modeling Stages}

In order to identify how the thinking process progresses over the time of taking the exercise, timeline plots have been drawn for each modeler's protocol. Due to space limitations only the timeline plot for modelers 2 and 6 are provided here (Figure 3 and 4). Overall, expert modelers follow a similar pattern throughout the model development process. Observing the plots it becomes apparent that modelers frequently switched their attention among topics.

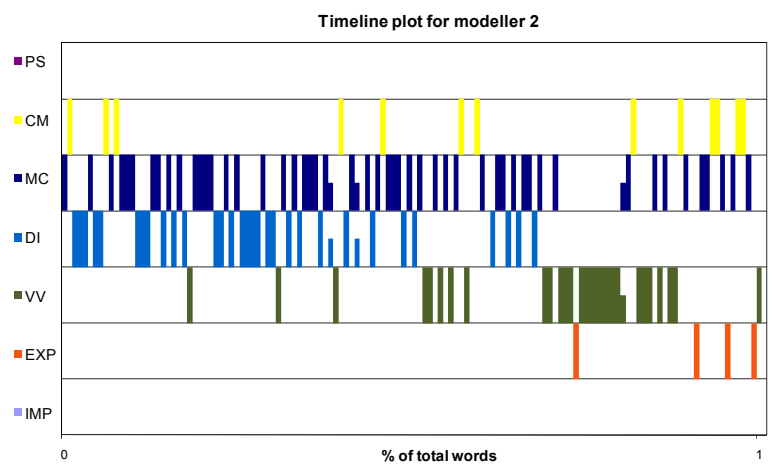

Figure 3: Timeline plot for expert modeler 2

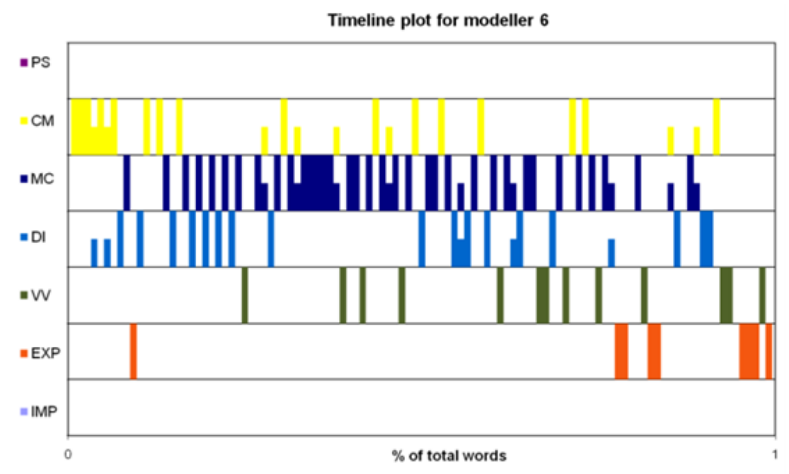

Figure 4: Timeline plot for expert modeler 6

Looking more specifically into the sequence of occurrence of each modeling topic, problem structuring, even though attended to only by three modelers (modelers 1, 3 and 5), it was generally attended to at the beginning of the protocols. Attention to conceptual modeling appears at various points throughout the modeling task. Only modeler 3 has a concentration of bars for conceptual modeling mostly at the first half 
of the protocol. High density of bars for model coding is obvious in all timelines, which appear more often as the task progresses. Data inputs is a topic well attended to by all expert modelers. All modelers enter the data in the model during the first half or at the middle of the modeling task. Verification \& validation of the model is attended to after some part of the model has been created and some data entered. Verification \& validation can follow two routes. One route follows the following sequence, the whole model is created and data entered and then the model is verified or validated (modeler 1 and 2). Alternatively, smaller parts of the model are created and data entered and then the model validated, and then more parts of the model are created and data entered and then again validated, and so on (modelers 4, 5, and 6). Results \& experimentation as well as implementation have not been attended to extensively by participating modelers. However, these tend to be attended to at the end of the protocol, with the exception of Modeler 3 only, who attended to results \& experimentations and implementation at the beginning of the protocol instead of after model building.

The timeline plot for Modeler 3 was somewhat different compared to other modelers as the model was not completely finished, to enable the modeler to consider experimentation and implementation again. Nevertheless, the protocol was still considered appropriate because the pattern of attention overall fits the pattern of the other six modelers, who still paid little attention to the last two stages. Therefore, this protocol is included in the sample.

\subsection{Pattern of Iterations Between Modeling Topics}

The iterations among modeling topics is explored using transition matrices, with a view to further understand the pattern of iterations followed by the DES modelers. A transition matrix represents the crosstabulation of the sequence of attention between successive pairs of episodes in a protocol. The transitions among topics for selected modelers, modelers 2, 3 and 6 are displayed in figure 5. The cells in the transition matrices have been highlighted according to the number of transitions counted, with the view to identifying the dominance of modelers' thinking. Based on the legend displayed, the darkest shades represent the transitions that occur most frequently, indicating the dominant loop of modelers' thinking.

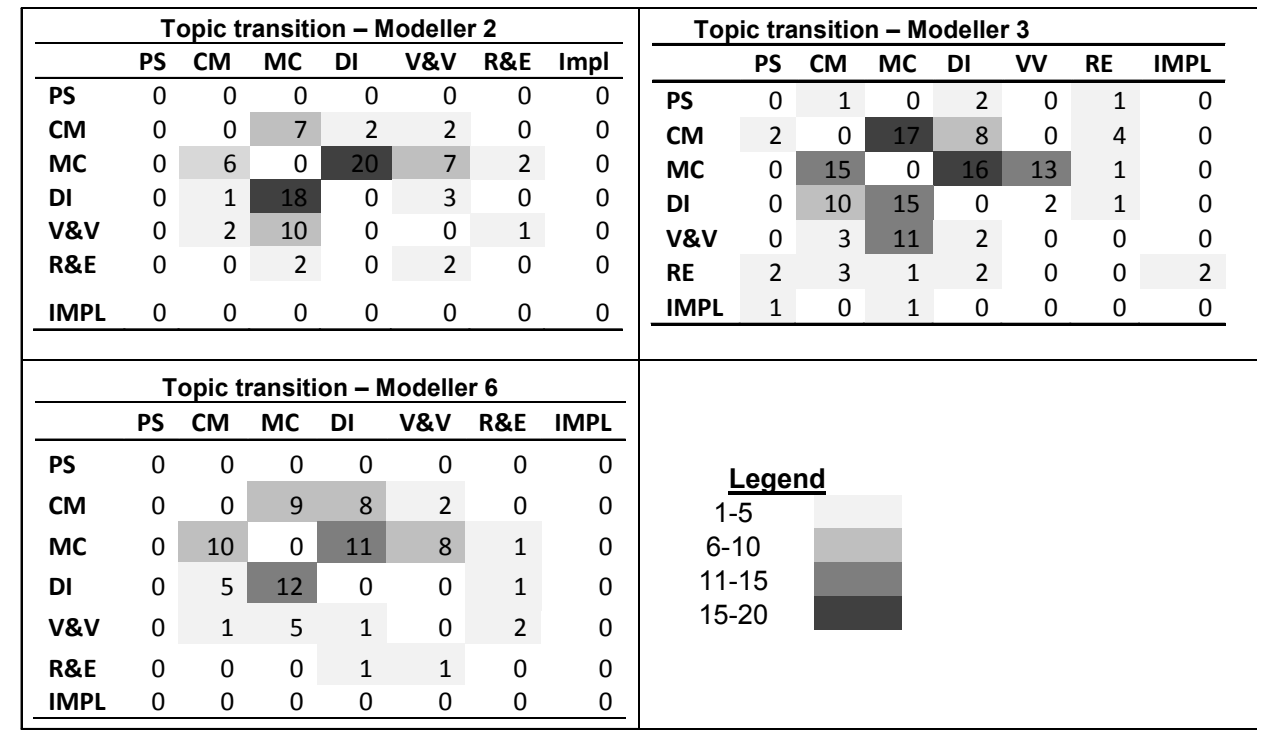

Figure 5: Transition matrices for modelers 2, 3 and 6

Obviously the number of transitions is proportionate to the total number of episodes identified for each protocol and hence the density of the transitions should be taken into consideration. Table 3 displays the total number of transitions per modeler as a proportion of the total number of episodes. The highest proportion of iterations in a ranked order starting from the highest corresponds to modelers $6(72 \%), 2$ 
$(63 \%)$ and $4(63 \%)$, with smaller proportions ranging around the $50 \%$ mark for the rest of the modelers. This sub-group does not seem to have any unique characteristics that distinguish them from the rest of the modelers in terms of length of experience, education on simulation and/or software used (Table 1). Higher transitions have been associated with better and more iterative design processes (Atman et al. 1999; Willemain and Powell 2006; Wang and Brooks 2007). However, a 72\% level of transitions undertaken by modeler 6 with 8 years experience could be considered considerably high. A clear association between transitions and length of experience cannot be established. This could suggest that the level of iterations in modeling activity does not seem to be associated with modellers' length of experience, and it perhaps seems to be down to the individual modeler's style. Nevertheless, it should be borne in mind that participants in this study were all expert modelers with at least 4 years of experience in modeling. If these proportions were compared to equivalent ones by novice modelers, lower proportions would be expected.

Table 3: Transition of attention expressed as a proportion of the total number of episodes in a protocol.

\begin{tabular}{|l|c|c|c|}
\hline & $\begin{array}{c}\text { Number of } \\
\text { transitions }\end{array}$ & $\begin{array}{c}\text { Total } \\
\text { episodes }\end{array}$ & $\begin{array}{c}\text { Proportion } \\
\text { of transitions }\end{array}$ \\
\hline Modeler 1 & 66 & 119 & $55 \%$ \\
\hline Modeler 2 & 85 & 135 & $63 \%$ \\
\hline Modeler 3 & 136 & 297 & $46 \%$ \\
\hline Modeler 4 & 77 & 122 & $63 \%$ \\
\hline Modeler 5 & 141 & 286 & $49 \%$ \\
\hline Modeler 6 & 78 & 109 & $72 \%$ \\
\hline
\end{tabular}

Observing the individual transition matrices for each modeler, it is obvious that the thinking process dominates among the following topics: model coding, data inputs, in most cases progressing to verification \& validation. For some modelers conceptual modeling is also included in the dominant loop. Two different styles of modeling activity can be identified based on the spread of transitions. For modelers 1 and 2, the dominant loop concentrates on model coding and data inputs and to a lesser extent on the rest of the modeling topics. A similar pattern is followed by modeler 4, adding into the loop verification and validation. The second group of modelers $(3,5$ and 6$)$ have a higher spread of transitions between conceptual modeling, model coding, data inputs and verification \& validation.

\section{DISCUSSION AND CONCLUSIONS}

The analysis undertaken in this study confirms that model coding is the most frequented topic that the six expert modelers return to most often. This is consistent with the estimates reported by Hollocks (2001). However, the equivalent experimentation and post-experimentation activities were attended to significantly less in this study. This could be mainly explained due to the lack of a real life client and the laboratory nature of the exercise, hence the modelers did not spend time on reporting the results or considering scenarios, which would have been the case in a real life simulation project.

The participating modelers alternate most often among 3 modeling topics: model coding, data inputs and verification \& validation (shown by the darker highlighted cells in the transition matrix (Figure 5)). These transitions determine the dominant loop in modelers' thinking process. In terms of the pattern of transitions in the modeling process, the expert modelers start initially with conceptual modeling, model coding and data inputs, while later on in the protocol the thinking process progresses to the other modeling topics, such as verification and validation or model results.

It was furthermore observed that most expert modelers undertook conceptual modeling at the same time as model coding. They were most of the time undertaking these two aspects of modeling simultaneously. This is shown by the high number of transitions between the two topics, but also in some cases by bars shared half and half between model coding and conceptual modeling as it was felt that these episodes 
referred to both topics. The main reason for this observation could be that the expert modelers were confident with modeling at the same time as conceptualizing, but also due to the "easier to use" simulation software that makes it possible for one to conceptualize at the same time as modeling on the computer.

Obviously, it should be noted that the analysis of the protocols was based on the researcher's interpretation of participants' verbalizations. Hence, some level of subjectivity is present in the results of the study. A different researcher might have reached different conclusions (using a different coding scheme with different definitions). To minimize the effect of subjectivity, the protocols were coded 3 times, involving in one case a third party. A bigger sample size could have also provided more representative results, however, due to project timescales this was not feasible. For future research, a larger sample size and alternate case studies could be used with the view to exploring in more depth the differences in modeling styles observed. Furthermore a wider sample base, involving expert modelers from other countries, US, Europe, etc. could be used to identify differences in modeling behavior due to culture differences

With reference to the data (verbal protocols) collection process, the modeling sessions were implemented as part of artificial laboratory settings, where the modelers at times felt the pressure of time or the pressure of being observed. The task given to the participants was a simple and a quite structured task to ensure completion of the exercise for a limited amount of time. These factors have to some extent affected the smaller amount of verbalizations for modeling topics such as: problem structuring, results \& experimentation and implementation.

The study presented takes an empirical approach to exploring the model development process in simulation as followed by expert modelers in the field. The modeling process is analyzed by undertaking a quantitative analysis of the thought processes, based on the modeling activities or topics, that expert modelers think about during a modeling exercise. This study provides empirical evidence about the modeling processes followed by practitioner modelers in discrete-event simulation, which can be used in teaching novices the art of simulation modeling. Differences in the modeling activity undertaken by the expert modelers are observed, which are believed to be a result of modelers' individual modeling style. The differences could not be attributed to typical modeler characteristics, such as level of experience, education or software used as there was not a clear association. Future work could extend this study using a larger sample of expert and perhaps novice modelers in order to identify typical modeling styles.

\section{ACKNOWLEDGMENTS}

The author would like to thank Stewart Robinson for the help and support provided during the study. Many thanks go to the modelers who participated in the experiment and to Suchi Collingwood for the help in blind checking the protocols in the third round of the coding process.

\section{REFERENCES}

Arthur, J. D., and R. E. Nance. 2007. "Investigating the Use of Software Requirements Engineering Techniques in Simulation Modelling." Journal of Simulation 1(3): 159-174.

Atman, C. J., J. R. Chimka, K. M. Bursic, and H. L. Nachtmann. 1999. "A Comparison of Freshman and Senior Engineering Design Processes." Design Studies 20(2): 131-152.

Balci, O., and R. E. Nance. 1987. "Simulation Model Development Environments: A Research Prototype." The Journal of the Operational Research Society 38(8): 753-763.

Banks, J., J. S. Carson, B. L. Nelson, and D. M. Nicol. 2001. Discrete-Event System Simulation. Upper Saddle River, New Jersey: Prentice Hall.

Cochran, J. K., G. T. Mackulak, and P. A. Savory. 1995. "Simulation Project Characteristics in Industrial Settings." Interfaces 25(4): 104-113.

Ericsson, K. A., and H. A. Simon. 1984. Protocol Analysis: Verbal Reports as Data. Boston: The MIT Press.

Grove, P., J. Macleod, and D. Godfrey. 1998. "Forecasting the Prison Population." OR Insight 11(1): 3-9. 
Hollocks, B. 2001. "Discrete-Event Simulation: An Enquiry into User Practice." Simulation Practice and Theory 8: 451-471.

Law, A. M. 2007. Simulation Modeling and Analysis. Boston, London: McGraw-Hill.

Nance, R. E. 1987. "The Conical Methodology: A Framework for Simulation Model Development." Technical Report TR-87-08, Computer Science, Virginia Polytechnic Institute and State University,

Nance, R. E. 1994. "The Conical Methodology and the Evolution of Simulation Model Development." Annals of Operations Research 53(1):1-45.

Pidd, M. 1999. "Just Modeling Through: A Rough Guide to Modeling." INTERFACES 29(2): 118-132.

Pidd, M. 2004. Computer Simulation in Management Science. Chichester:Wiley.

Robinson, S. 2004. Simulation: The Practice of Model Development and Use. Chichester:Wiley.

Robinson, S. 2005. "Discrete-Event Simulation: From the Pioneers to the Present, What Next?" Journal of the Operational Research Society 56(6): 619-629.

Sanchez, P. J. 2007. "Fundamentals of Simulation Modeling." In Proceedings of the 2007 Winter Simulation Conference, edited by S. G. Henderson, B. Biller, M.-H Hsieh, J. Shortle, J. D. Tew, and R. R. Barton, 54-62. Piscataway, New Jersey: Institute of Electrical and Electronics Engineers, Inc.

Sargent, R. G. 2010. "Verification and Validation of Simulation Models." In Proceedings of the 2010 Winter Simulation Conference, edited by B. Johansson, S. Jain, J. Montoya-Torres, J. Hugan, and E. Yücesan, 166-183. Piscataway, New Jersey: Institute of Electrical and Electronics Engineers, Inc.

Shannon, R. E. 1975. Systems Simulation: The Art and Science. Englewood Cliffs, NJ: Prentice Hall.

Shannon, R. E. 1998. "Introduction to the Art and Science of Simulation." In Proceedings of the 1998 Winter Simulation Conference, edited by D. J. Medeiros, E. F. Watson, J. S. Carson, and M. S. Manivannan, 7-14. Piscataway, New Jersey: Institute of Electrical and Electronics Engineers, Inc.

Tako, A. A. 2009. "Development and Use of Simulation Models in Operational Research: A Comparison of Discrete-Event Simulation and System Dynamics." Ph.D. Thesis. Warwick Business School. University of Warwick, Coventry, UK..

Tako, A. A., and S. Robinson. 2010. "Model Development in Discrete-Event Simulation and System Dynamics: An Empirical View." European Journal of Operational Research 207: 784-794.

Todd, P., and I. Benbasat. 1987. "Process Tracing Methods in Decision Support Systems Research: Exploring the Black Box." MIS Quarterly 11(4): 493-512.

Wang, W., and R. J. Brooks. 2007. "Improving the Understanding of Conceptual Modelling." Journal of Simulation 1(3): 153-158.

Willemain, T. R. 1994. "Insights on Modeling from a Dozen Experts." Operations Research 42(2): 213222.

Willemain, T. R. 1995. "Model Formulation: What Experts Think About and When." Operations Research 43(6): 916-932.

Willemain, T. R., and S. G. Powell 2006. "How Novices Formulate Models. Part II: A Quantitative Description of Behaviour." The Journal of the Operational Research Society 58(10): 1271-1283.

\section{AUTHOR BIOGRAPHIES}

ANTUELA A. TAKO is a Lecturer in Operations Research at Loughborough University. She holds a $\mathrm{PhD}$ in Simulation and an MSc in Management Science and Operational Research from the University of Warwick. Her research focuses on the comparison of simulation approaches (Discrete-Event Simulation and System Dynamics), participative simulation modeling and conceptual modeling. Her email address is a.takou@lboro.ac.uk. 